\title{
In vitro and in vivo evaluation of ordered mesoporous silica as a novel adsorbent in liquisolid formulation
}

This article was published in the following Dove Press journal:

International Journal of Nanomedicine

12 January 2012

Number of times this article has been viewed

\section{Bao Chen ${ }^{1,2}$ \\ Zhouhua Wang' \\ Guilan Quan' \\ Xinsheng Peng' \\ Xin Pan' \\ Rongchang Wang' \\ Yuehong $\mathrm{Xu}{ }^{\prime}$ \\ Ge Li ${ }^{2}$ \\ Chuanbin $\mathrm{Wu}^{1,2}$}

'School of Pharmaceutical Sciences, ${ }^{2}$ Research and Development Center of Pharmaceutical Engineering,

Sun Yat-sen University, Guangzhou,

People's Republic of China
Correspondence: Chuanbin Wu School of Pharmaceutical Sciences, Sun Yat-sen University, Guangzhou, 510006, People's Republic of China Email cbwu2000@yahoo.com
Background: A liquisolid technique has been reported to be a new approach to improve the release of poorly water-soluble drugs for oral administration. However, an apparent limitation of this technique is the formulation of a high dose because a large amount of liquid vehicle is needed, which finally results in a low-dose liquisolid formulation. Silica as an absorbent has been used extensively in liquisolid formulations. Although nanoparticle silica can be prepared and used to improve liquid adsorption capacity, loading a high dose of drug into a liquisolid is still a challenge. With the aim of improving adsorption capacity and accordingly achieving high drug loading, ordered mesoporous silica with a high surface area and narrow pore size distribution was synthesized and used in a liquisolid formulation.

Methods: Ordered mesoporous silica was synthesized and its particle size and morphology were tailored by controlling the concentration of cetyltrimethyl ammonium bromide. The ordered mesoporous silica synthesized was characterized by transmission electron microscopy, scanning electron microscopy, Fourier transform infrared spectroscopy, small-angle x-ray diffraction, wide angle x-ray diffraction, and nitrogen adsorption-desorption measurements. The liquid adsorption capacity of ordered mesoporous silica was subsequently compared with that of conventional silica materials using PEG400 as the model liquid. Carbamazepine was chosen as a model drug to prepare the liquisolid formulation, with ordered mesoporous silica as the adsorbent material. The preparation was evaluated and compared with commercially available fast-release carbamazepine tablets in vitro and in vivo.

Results: Characterization of the ordered mesoporous silica synthesized in this study indicated a huge Brunauer-Emmett-Teller surface area $\left(1030 \mathrm{~m}^{2} / \mathrm{g}\right)$, an ordered mesoporous structure with a pore size of $2.8 \mathrm{~nm}$, and high adsorption capacity for liquid compared with conventional silica. Compared with fast-release commercial carbamazepine tablets, drug release from the liquisolid capsules was greatly improved, and the bioavailability of the liquisolid preparation was enhanced by $182.7 \%$.

Conclusion: Ordered mesoporous silica is a potentially attractive adsorbent which may lead to a new approach for development of liquisolid products.

Keywords: ordered mesoporous silica, poorly water-soluble drug, carbamazepine, liquisolid, bioavailability

\section{Introduction}

In recent years, the liquisolid technique has been reported as a new approach to improve the rate of release of poorly water-soluble drugs intended for oral administration. ${ }^{1-7}$ With this technique, liquid formulations, such as solutions or suspensions of waterinsoluble solid drugs in nonvolatile liquid vehicles, can be converted into free-flowing and readily compressible powders by blending with selected powder excipients, 
ie, carrier and coating materials. The advantages of this technique include process simplicity, low cost, and controlled drug release. ${ }^{8,9}$ However, an apparent limitation of this technique is the formulation of high doses because a large amount of liquid vehicle is needed, and accordingly, a large amount of carrier and coating materials needs to be used, which results in low-dose drug formulations.

Silica powder has been widely used as a coating material in liquisolid formulations for liquid adsorption due to its large specific surface area, and nontoxic and inert characteristics. ${ }^{10}$ Although nanoparticle formulations of silica, such as Aerosil ${ }^{\circledR} 200$ and $\mathrm{HDK}^{\circledR} \mathrm{N} 20 \mathrm{P}$ with a surface area of $200 \mathrm{~m}^{2} / \mathrm{g}$ were prepared and used to improve liquid adsorption capacity, loading high doses of drugs in a liquisolid is still a challenge.

First reported by Mobil scientists, ${ }^{11,12}$ mesoporous silica has attracted considerable attention as a molecular sieve due to its inherent properties, such as a large pore volume and surface area, and a tunable narrow pore size distribution. ${ }^{13,14}$ These properties suggest that mesoporous silica may have a higher liquid adsorption capacity when used in liquisolid formulations as compared with conventional silica. To confirm this assumption, ordered mesoporous silica was synthesized in the present study using a surfactant-directing method. In previous research, many methods had been reported for control of the morphology and particle size of mesoporous silica by varying the parameters of synthesis, including temperature, ${ }^{15} \mathrm{pH}$ value, ${ }^{16}$ cosolvent, ${ }^{17}$ and cosurfactant. ${ }^{18}$ In the present study, particle size and morphology of ordered mesoporous silica was explored by controlling the concentration of surfactant. The ordered mesoporous silica synthesized was characterized by small-angle x-ray diffraction, wide angle $\mathrm{x}$-ray diffraction, scanning electron microscopy, transmission electron microscopy, Fourier transform infrared spectroscopy, and nitrogen adsorption-desorption measurements. The liquid adsorption capacity of ordered mesoporous silica was subsequently compared with that of conventional silica materials using PEG400 as a model liquid.

Carbamazepine (5H-dibenzazepine-5-carboxamide) is classified as a class II drug (low solubility and high permeability) according to the biopharmaceutic classification system. ${ }^{19}$ This high-dose drug ( $\geq 100 \mathrm{mg}$ ) is also known to have four anhydrous polymorphs, and these polymorphs may affect drug release and bioavailability. ${ }^{20,21}$ Therefore, carbamazepine was chosen as a model drug to prepare a liquisolid formulation with ordered mesoporous silica as the adsorbent material. The preparation was evaluated and compared with commercially available fast-release carbamazepine tablets in vitro and in vivo.

\section{Materials and methods Materials}

Carbamazepine was purchased from Suzhou Hengyi Pharmaceuticals Co, Ltd (Jiangsu, China). Commercially available fast-release carbamazepine $100 \mathrm{mg}$ tablets were purchased from Guangdong Huanan Pharmacy Ltd (Guangdong, China). Starch 1500, microcrystalline cellulose (Vivapur 12), and $\mathrm{HDK}^{\circledR} \mathrm{N} 20 \mathrm{P}$ silica (Wacker, Munich, Germany) were kindly donated by Jiefu Trade Co, Ltd (Guangzhou, China). Microsilica was obtained from Huzhou Zhanwang Pharmaceutical Co, Ltd (Zhejiang, China). Standard silica (99.999\%) was obtained from Shanghai Reagent Factory (Shanghai, China). PEG 400 was purchased from Sigma-Aldrich (Hong Kong, China). Cetyltrimethyl ammonium bromide (CTAB), tetraethoxysilane, and ammonia were obtained from Fuchen Chemicals Reagent Factory (Tianjin, China). High-pressure liquid chromatography (HPLC)-grade acetonitrile was obtained from Fisher Scientific (Beijing, China). Other chemicals were of reagent grade and used without further purification.

\section{Ordered mesoporous silica synthesis}

Ordered mesoporous silica was synthesized in alkaline media using modified literature recipes. ${ }^{22}$ Typically, $5 \mathrm{~g}$ of CTAB was dissolved in $520 \mathrm{~mL}$ of $15 \%(\mathrm{w} / \mathrm{w})$ ammonia at $60^{\circ} \mathrm{C}$ under constant stirring at $200 \mathrm{rpm}$ for 0.5 hours. Then $20 \mathrm{~mL}$ of tetraethoxysilane was added dropwise into the solution under continuous stirring for 2 hours. After aging in a sealed container at $60^{\circ} \mathrm{C}$ for 24 hours without stirring, the mixture was filtered to recover the solid. The solid product was washed with water until the filtrate was neutral and then rinsed twice with alcohol before being dried overnight at $80^{\circ} \mathrm{C}$. Calcination of the solid product was carried out by slowly increasing the temperature from room temperature to $550^{\circ} \mathrm{C}$ within 4 hours and maintaining at $550^{\circ} \mathrm{C}$ for another 6 hours. At last, a pure white and feathery powder, ordered mesoporous silica, was obtained.

The synthesis of ordered mesoporous silica consists of a series of hydrolysis and condensation reactions of an alkoxide at the surface of CTAB micelles. The condensation reaction proceeds either via alcohol condensation or water condensation. The total reaction can be summarized as follows:

$$
\mathrm{nSi}\left(\mathrm{OC}_{2} \mathrm{H}_{5}\right)+2 \mathrm{nH}_{2} \mathrm{O}=\mathrm{nSiO}_{2}+4 \mathrm{nC}_{2} \mathrm{H}_{5} \mathrm{OH}
$$


To control the particle size and shape of ordered mesoporous silica, the same synthesis process described above was carried out by varying the concentration of CTAB in a range of $0.5 \%-3.0 \%(\mathrm{w} / \mathrm{w})$. The yield of ordered mesoporous silica was calculated according to the following formula:

$$
\mathrm{Y}(\text { yield })=\mathrm{W}_{\text {OMS }} /\left(\mathrm{W}_{\text {TEOS }} \times 28.4 \%\right) \times 100 \%
$$

where $\mathrm{W}_{\text {OMS }}$ and $\mathrm{W}_{\text {TEOS }}$ are the weight of ordered mesoporous silica collected after calcination and tetraethoxysilane, respectively.

\section{Characterization of ordered mesoporous silica}

Scanning electron microscopy measurements were carried out with a JSM-6330F microscope (JEOL, Tokyo, Japan). Samples were deposited on the surface of a silicon wafer by dropping a suspension of samples in ethanol which was presonicated for 5 minutes, and then sputter-coated for two cycles with gold. Transmission electron microscopy was performed by a lowexposure technique using a JEOL 2010 electron microscope with analySIS 5.0 software (Olympus, Münster, Germany) to reduce the influence of beam damage and sample drift. Samples for transmission electron microscopy measurements were prepared by dipping a carbon-coated copper grid into a suspension of sample in ethanol, which was presonicated for 5 minutes. The pore size and pore wall thickness were measured by analySIS 5.0 software, which can directly show the results after selecting the object of measurement.

Nitrogen adsorption-desorption isotherms were measured at $-196^{\circ} \mathrm{C}$ by ASAP $2020 \mathrm{C}$ (Micromeritics, Norcross, GA). Prior to analysis, the samples were degassed in a vacuum at $300^{\circ} \mathrm{C}$ for 3 hours. Pore volume was determined from the adsorption branch of nitrogen adsorption-desorption isotherm curve at a relative nitrogen pressure $\mathrm{P} / \mathrm{P}_{0}=0.975$ signal point. Pore diameter was calculated from the adsorption branch of the isotherms using the Barrett-Joyner-Halenda method, and specific surface area was calculated using the multiple-point Brunauer-Emmett-Teller method.

Fourier transform infrared spectra were obtained using a Fourier transform infrared spectrometer (Bruker, Germany) to scan over a spectral region of $400-4000 \mathrm{~cm}^{-1}$ on a thinly sliced sample compressed from the dry mixture of ordered mesoporous silica and $\mathrm{KBr}$.

Small-angle and wide-angle $\mathrm{x}$-ray diffraction patterns of ordered mesoporous silica were analyzed on a D/MAX 2200VPC diffractometer (Rigaku, Tokyo, Japan) using $\mathrm{CuK} \alpha$ radiation with $2 \theta$ in the range of $0.6^{\circ}-6^{\circ}$ and $10^{\circ}-40^{\circ}$, respectively.

\section{Evaluation of adsorption capacity with liquid}

PEG400 was used as a model liquid to evaluate the adsorption capacity of the silicas. Firstly, conventional silicas $\left(\mathrm{HDK}^{\circledR} \mathrm{N} 20 \mathrm{P}\right.$ silica and Microsilica) and ordered mesoporous silica were dried at $150^{\circ} \mathrm{C}$ for 2 hours in order to remove any residual moisture. Then $1.0 \mathrm{~g}$ of each silica sample was mixed with an adequate amount of PEG400. The mixture was sealed in a container for one hour to reach an adsorption equilibrium. Afterwards, the mixture was spread on a preweighed filter paper and subsequently sealed in a container overnight to remove the overloading of liquid from silicas by adsorbing any free liquid onto filter paper. Finally, the solid mixture was carefully removed and the filter paper was weighed accurately again. The adsorption capacity was calculated according to the following formula:

$$
\text { A }(\text { adsorption capacity })=\left[\mathrm{W}_{1}-\left(\mathrm{W}_{\mathrm{fl}}-\mathrm{W}_{\mathrm{f0}}\right)\right] / \mathrm{W}_{\mathrm{m}}
$$

where $\mathrm{W}_{1}$ and $\mathrm{W}_{\mathrm{m}}$ are the weight of liquid and silica material, respectively; $\mathrm{W}_{\mathrm{f} 0}$ and $\mathrm{W}_{\mathrm{fl}}$ represent, respectively, the original weight and final weight after removing materials of the filter paper.

\section{Angle of repose test}

In order to determine the appropriate ratio of carrier and coating material in the liquisolid system, the angle of repose was measured using a BT-1000 powder characteristics tester (BT, Dandong, China), which can directly read out the angle of repose of a sample without calculation.

\section{Preparation of liquisolid capsules}

Two liquisolid formulations, denoted as F1 and F2, were prepared using carbamazepine as the model drug and ordered mesoporous silica as follows. For F1, about $3 \mathrm{~g}$ of carbamazepine was dissolved in $40 \mathrm{~mL}$ of PEG400 to prepare the liquid medication. Then $15 \mathrm{~g}$ of a binary mixture of starch 1500 as the carrier and ordered mesoporous silica as the coating material at the ratio of $2: 1(\mathrm{w} / \mathrm{w})$, which was determined by the results of the angle of repose, was added to the liquid medication under continuous mixing to form a liquisolid system. Afterwards, $3 \mathrm{~g}$ of $10 \%$ starch slurry was added into the liquisolid system to obtain a wet powder mixture for granulation. The granules were produced through a 16 mesh sieve and dried in an oven at $40^{\circ} \mathrm{C}$ overnight. Finally, the dried granules were encapsulated into hard shell gelatin capsules. 
To investigate the influence of the carrier on drug release, another formulation, F2, was prepared using microcrystalline cellulose as the carrier instead of starch 1500 following the same preparing steps described above.

In addition, conventional silica, N20P, was used as a coating material to mix with starch 1500 as the carrier to prepare carbamazepine liquisolid capsules following the same preparing steps. However, due to the poor adsorption capacity of N20P, in order to obtain flowability similar to that of F1 granules, $30 \mathrm{~g}$ of a binary mixture of starch 1500 and $\mathrm{N} 20 \mathrm{P}$ at a ratio of 2:1 (w/w) was used to load the same amount of carbamazepine liquid. This control formulation was denoted as F3.

\section{In vitro dissolution test}

The dissolution test was carried out using a USP II paddle method $(75 \mathrm{rpm}, 900 \mathrm{~mL}$ of simulated gastric fluid as dissolution medium at $37^{\circ} \mathrm{C}$ ) with a ZRS-8G dissolution tester (TDTF, Tianjin, China). A sample equivalent to $100 \mathrm{mg}$ of carbamazepine was added to each vessel. At predetermined time intervals, $5 \mathrm{~mL}$ of dissolution medium was withdrawn, filtered through a $0.22 \mu \mathrm{m}$ Millipore filter, and assayed by ultraviolet absorption at a wavelength of $284 \mathrm{~nm}$. An equal amount of fresh medium was added to the vessel after each sample was drawn for compensation. Each dissolution test was performed in triplicate.

\section{Animal experiments}

All animal experimental procedures were approved by the animal ethics committee of Sun Yat-sen University in accordance with National Institute of Health and Nutrition Guidelines for the Care and Use of Laboratory Animals. Six healthy 1.5-2.0 year old female beagle dogs weighing 14-15 kg were used in a two-treatment, two-period, twosequence crossover design. Prior to each study, the dogs were fasted for 14-16 hours but allowed to drink water ad libitum. On each occasion, the dogs received the following oral formulations and $40 \mathrm{~mL}$ of water: one commercially available fast-release carbamazepine $100 \mathrm{mg}$ tablet, or four liquisolid capsules (F1) equivalent to a dose of $100 \mathrm{mg}$ carbamazepine. A washout period of at least two weeks was allowed between treatments.

Prior to drug administration and at 10,20,30,45, 60,75, 90, 120, 180, 240, 300, and 480 minutes after dosing, $4 \mathrm{~mL}$ blood samples were taken into heparinized tubes at each time point through the cephalic vein of the hind leg. Plasma was separated by centrifugation ( $3000 \mathrm{rpm}, 15$ minutes) and stored at $-20^{\circ} \mathrm{C}$ prior to analysis.

\section{Analysis of plasma carbamazepine concentration}

Carbamazepine was assayed using an HPLC method, which was validated following international guidelines. A $500 \mu \mathrm{L}$ plasma sample was mixed with $20 \mu \mathrm{L}$ of methanol containing an internal standard (pentobarbital, $100 \mu \mathrm{g} / \mathrm{mL}$ ) and vortexed for 30 seconds. The sample was then mixed with $2 \mathrm{~mL}$ of acetoacetate as the extraction solvent and vortexed for 3 minutes, followed by centrifugation at 10,000 rpm for 3 minutes to separate the layers. The supernatant was collected into clean centrifuge tubes by aspiration and evaporated under vacuum at room temperature for 2 hours. The evaporated sample was redissolved in $150 \mu \mathrm{L}$ of mobile phase, then $50 \mu \mathrm{L}$ of this test sample was injected for HPLC analysis.

The HPLC analysis was carried out on a Waters model 1525 pump equipped with a 2487 ultraviolet detector, a 717 autosample system, and Empower software. Carbamazepine separation was completed on a Symmetry ${ }^{\circledR} \mathrm{C}_{18}$ column $(4.6 \times 250 \mathrm{~mm}, 5 \mu \mathrm{m})$ with a guard column $(4.6 \times 12.5 \mathrm{~mm}$, $5 \mu \mathrm{m})$ using a mobile phase consisting of acetonitrile and water $(40: 60, \mathrm{v} / \mathrm{v})$ at a flow rate of $1.0 \mathrm{~mL} /$ minute. The detection wavelength was set at $230 \mathrm{~nm}$. The column working temperature was kept at $40^{\circ} \mathrm{C}$, and the injection volume was $50 \mu \mathrm{L}$. Under these conditions, carbamazepine and pentobarbital eluted at approximately 8 minutes and 9 minutes, respectively. A linear standard curve was generated $\left(r^{2}>0.99\right)$ over the examined carbamazepine concentration range of $0.05-3.0 \mu \mathrm{g} / \mathrm{mL}$. With a limit of quantitation of $0.05 \mu \mathrm{g} / \mathrm{mL}$, the accuracy of the assay was $90 \%-110 \%$, and the interday and intraday precisions in terms of coefficients of variation were all less than $10 \%$. Carbamazepine recoveries for three different concentrations $(0.1,1.0$, and $3.0 \mu \mathrm{g} / \mathrm{mL})$ were $99.1 \% \pm 1.6 \%, 100.8 \% \pm 4.0 \%$, and $98.0 \% \pm 2.2 \%$, respectively.

\section{Pharmacokinetic and statistical analyses}

The peak plasma concentration $\left(\mathrm{C}_{\max }\right)$ and the time to reach the peak plasma concentration $\left(\mathrm{T}_{\text {max }}\right)$ were obtained directly from the individual plasma concentration-time data. The area under the plasma concentration-time curve $\left(\mathrm{AUC}_{0-\infty}\right)$ was calculated by the trapezoidal rule from time 0 to infinity. The elimination constant $\left(\mathrm{K}_{\mathrm{e}}\right)$ was estimated from the elimination segment of the curve, as the slope of logarithm concentration-time plot, while the half-time $\left(\mathrm{t}_{1 / 2}\right)$ was calculated as $0.693 / \mathrm{K}_{\mathrm{e}}$. The relative bioavailability was calculated as $\left[\left(\mathrm{AUC}_{\text {test }} \times \mathrm{D}_{\text {ref }}\right) /\left(\mathrm{AUC}_{\text {ref }} \times \mathrm{D}_{\text {test }}\right)\right] \times 100$, where $\mathrm{D}$ is the dose, and "test" and "ref" correspond to the 
liquisolid formulation and commercial tablet, respectively. For statistical evaluation, analysis of variance was used to assess the significance of the difference between the pharmacokinetic parameters obtained.

\section{Results and discussion Synthesis of ordered mesoporous silica}

In the present study, ordered mesoporous silica of different morphology and particle size was synthesized by varying the concentration of the surfactant, CTAB. As shown in Figure 1, approximately spherical, rod-like, and curled rod-like mesoporous silicas were obtained. All particles were well monodispersed with a smooth surface, even after calcination at $550^{\circ} \mathrm{C}$ for 6 hours. With increasing concentrations of CTAB, it was noticed that the ordered mesoporous silica particles elongated in one direction, resulting in an increase in particle size.

On the other hand, it was noticed that lowering the CTAB concentration reduced the yield of ordered mesoporous silica remarkably. As shown in Table 1, the yield of ordered mesoporous silica with $0.5 \%$ CTAB was less than $50 \%$, while the yield of ordered mesoporous silica with $0.8 \%$ CTAB was more than $80 \%$. This may be due to CTAB, a structure-directing template, promoting the hydrolysis of an alkoxide. During hydrolysis and condensation of an alkoxide, bromide ions of CTAB are exchanged for silicate anions at the CTAB micelle surface, ${ }^{23,24}$ which leads to rapid hydrolysis of the alkoxide, resulting in complete formation of ordered mesoporous silica. However, in the case of $0.5 \%$ CTAB, too little CTAB may not be able to speed up the hydrolysis of alkoxide, resulting in incomplete formation and a low yield of ordered mesoporous silica.

In addition, the adsorption capacity of ordered mesoporous silica synthesized with different concentrations of CTAB is compared in Table 2. Only ordered mesoporous silica synthesized with $0.5 \%$ CTAB showed slightly higher adsorption capacity than the other formulations. However, the $P$ values were all $>0.05$, indicating that the differences in adsorption capacity were not statistically significant between ordered mesoporous silica synthesized with $0.5 \% \mathrm{CTAB}$ and the other formulations.

As a result, when the concentration of CTAB was in the range of $0.8 \%-3.0 \%$, the adsorption capacity and yield of ordered mesoporous silica did not show significant differences. However, it has been reported that CTAB has marked cytotoxicity. ${ }^{25}$ Therefore, the formulation with the lowest concentration of CTAB, ie, $0.8 \%$, was selected for further study.

\section{Characterization of ordered mesoporous silica}

The scanning electron images in Figure 1B show that the ordered mesoporous silica particles were rod-like in shape with a hexagonal cross-section (pointed to by arrows in Figure 1B). The transmission electron microscopic image of ordered mesoporous silica (Figure 1F) shows bright and dark areas which correspond to pores and silica walls, respectively. This confirms the hexagonal structure of ordered mesoporous
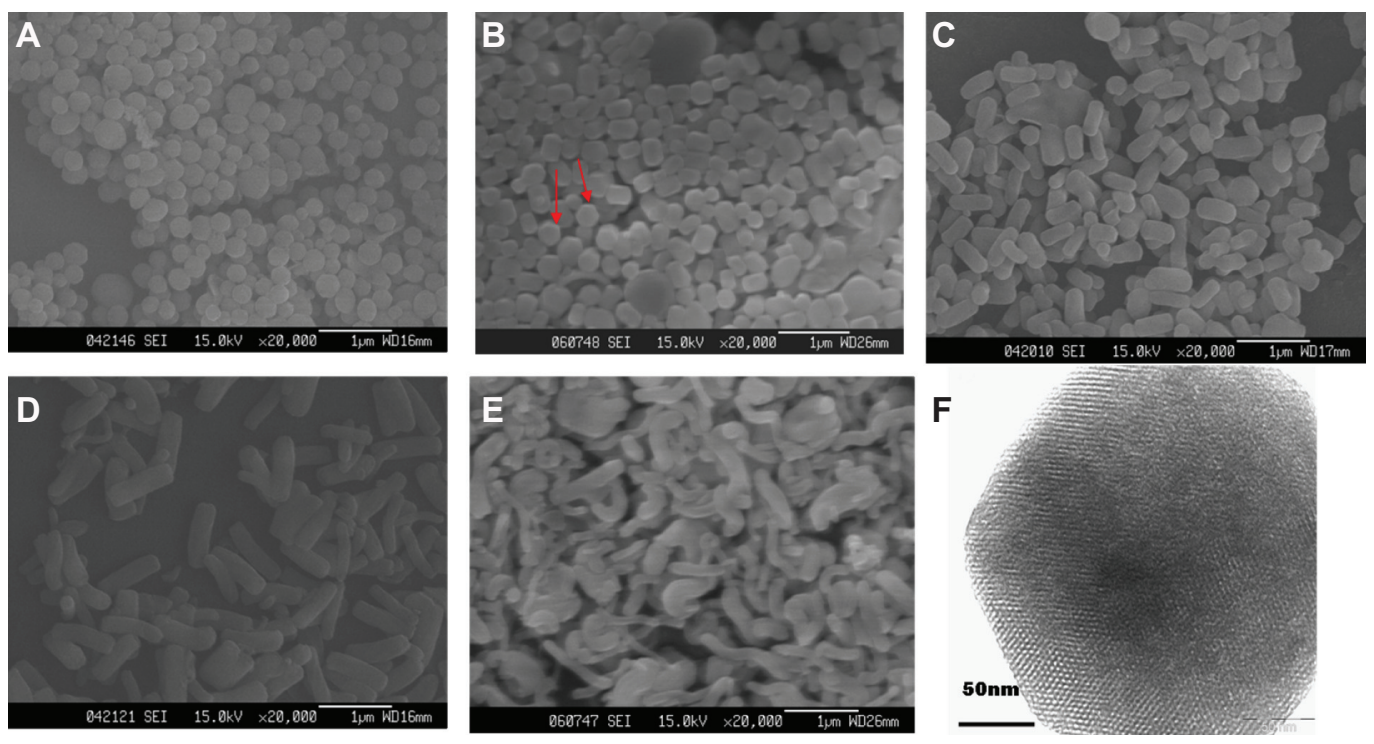

Figure I Scanning electron microscopic images of ordered mesoporous silica synthesized with different concentrations of cetyltrimethyl ammonium bromide (w/w). (A) $0.5 \%$, (B) $0.8 \%$, (C) 1.0\%, (D) 1.5\%, and (E) 3\%; and (F) transmission electron microscopic image of ordered mesoporous silica with $0.8 \%$ cetyltrimethyl ammonium bromide. 
Table I Particle size and yield of OMS synthesized with different concentrations of CTAB

\begin{tabular}{llllll}
\hline CTAB Concentration (\%, w/w) & $\mathbf{0 . 5}$ & $\mathbf{0 . 8}$ & I.0 & I.5 & 3.0 \\
\hline Particle shape & Near spheres & Rod-like & Rod-like & Rod-like & Cured rod-like \\
Mean particle size (nm) & D: 280 & D: 300 & D: 260 & D: 280 & D: 200 \\
& & L: 400 & L: 510 & L: 850 & L: 1000 \\
Yield $(\%)$ & 42.3 & 89.4 & 90.5 & 91.3 & 90.2 \\
\hline
\end{tabular}

Abbreviations: D, diameter; L, length; CTAB, cetyltrimethyl ammonium bromide; OMS, ordered mesoporous silica.

silica and further illustrates well-ordered mesopores with an average pore size of about $2.8 \mathrm{~nm}$, as measured by analySIS 5.0 software.

In order to obtain more precise information about the structure of mesoporous silica, nitrogen adsorptiondesorption measurements were performed. Figure 2 shows the nitrogen adsorption-desorption isotherms for ordered mesoporous silica which demonstrate a type IV isotherm with a typical capillary condensation step in the mesopores at a relative nitrogen pressure $\mathrm{p} / \mathrm{p}_{\mathrm{o}}$ ranging from 0.25 to 0.4 . This indicates that the samples possessed typical mesostructural ordering and a relatively uniform pore size distribution. ${ }^{26}$ The corresponding pore size distribution (Figure 3) calculated from the adsorption branch of the isotherms showed a narrow distribution of pore size in the range of $2.0-3.3 \mathrm{~nm}$. The mean Brunauer-Emmett-Teller surface area, pore volume, and pore size were calculated as $1030 \mathrm{~m}^{2} / \mathrm{g}, 0.81 \mathrm{~cm}^{3} / \mathrm{g}$, and $2.8 \mathrm{~nm}$, respectively. These findings imply a high adsorption capacity for ordered mesoporous silica. Further, the calculated mean pore size was consistent with the value measured on transmission electron microscopy images. However, the nitrogen adsorption-desorption isotherms for the two conventional silicas, ie, Microsilica and N20P, were quite different from that for ordered mesoporous silica (Figure 2). The isotherms for the conventional silicas are type III according to the International Union of Pure and Applied Chemistry classification, which is characteristic of nonporous or macroporous solids. ${ }^{27}$ Their corresponding pore size distribution also showed the absence of peaks in the range of $2-50 \mathrm{~nm}$. The Brunauer-Emmett-Teller surface areas of Microsilica and N20P were $78 \mathrm{~m}^{2} / \mathrm{g}$ and $194 \mathrm{~m}^{2} / \mathrm{g}$, respectively.

The small-angle $\mathrm{x}$-ray diffraction pattern of ordered mesoporous silica (Figure 4A) showed three well resolved peaks which were indexed as (100), (110), and (200) reflections associating with $\mathrm{P} 6 \mathrm{~mm}$ hexagonal symmetry, and this suggests a good long-range order of pores in ordered mesoporous silica. Based on the formula $a_{0}=(2 / \sqrt{3})$ $d_{100}{ }^{28}$, the lattice parameter $a_{0}$ was calculated as $4.32 \mathrm{~nm}$. Considering the pore wall thickness of 1-2 nm, as measured in Figure 1F, the pore size of ordered mesoporous silica was calculated as $2-3 \mathrm{~nm}$ by subtracting the pore wall thickness from $a_{0}$, and this was in agreement with the above measurements.

The wide angle $\mathrm{X}$-ray diffraction pattern (Figure 4B) confirmed that the ordered mesoporous silica was amorphous silicon dioxide, on account of a broad peak ranging from $2 \theta$ of $15^{\circ}$ to $30^{\circ}$, and this result is consistent with the published literature. ${ }^{29}$

CTAB has been used in research as a mesoporous template during ordered mesoporous silica synthesis and removed by post-synthesis calcination. However, CTAB was reported to have marked cytotoxicity, ${ }^{25}$ and to ensure CTAB was completely removed by calcination, Fourier transform infrared spectroscopy detection was carried out. As shown in Figure 5, the spectrum of CTAB showed two intense peaks at $2800-3200 \mathrm{~cm}^{-1}$, which were assigned to the asymmetric $\left(2918 \mathrm{~cm}^{-1}\right)$ and symmetric $\left(2849 \mathrm{~cm}^{-1}\right)$ stretching vibrations of $\mathrm{C}-\mathrm{CH}_{2}$ in the methylene chains. ${ }^{30}$ These peaks were observed in as-synthesized ordered mesoporous silica but absent in calcined ordered mesoporous silica, indicating that $\mathrm{CTAB}$ was completely removed by calcination. In the region of 400-1800 $\mathrm{cm}^{-1}$, characteristic peaks of silica were observed in as-synthesized ordered mesoporous silica and calcined ordered mesoporous silica, as well as in standard silica, indicating ordered mesoporous silica was pure silica and its chemical structure was intact, even after calcination at $550^{\circ} \mathrm{C}$ for 6 hours.

Table 2 Adsorption capacity of OMS synthesized with different concentrations of CTAB $(n=3)$

\begin{tabular}{llllll}
\hline CTAB Concentration (\%, w/w) & $\mathbf{0 . 5}$ & $\mathbf{0 . 8}$ & $\mathbf{I . 0}$ & $\mathbf{I . 5}$ & $\mathbf{3 . 0}$ \\
\hline Adsorption capacity $(\mathrm{g} / \mathrm{g})$ & $7.04 \pm 0.22$ & $6.87 \pm 0.30$ & $6.75 \pm 0.30$ & $6.56 \pm 0.24$ & $6.60 \pm 0.27$ \\
$P$ value* & & 0.36 & 0.33 & 0.10 & 0.15 \\
\hline
\end{tabular}

Note: *P values were calculated between OMS with $0.5 \%$ CTAB and the other OMS formulations.

Abbreviations: $\mathrm{CTAB}$, cetyltrimethyl ammonium bromide; OMS, ordered mesoporous silica. 


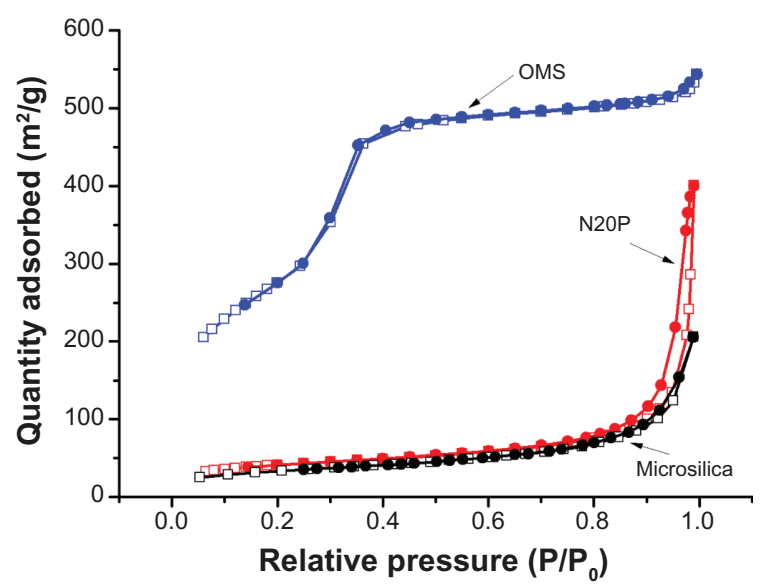

Figure 2 Nitrogen adsorption ( $\square$ )-desorption $(\bullet)$ isotherms of ordered mesoporous silica, Microsilica, and N20P.

\section{Adsorption capacity}

The above characterization results indicate that the synthesized ordered mesoporous silica was quite different from conventional silicas with regard to large pore volume, a huge surface area, and ordered mesoporous structure. These properties implied that ordered mesoporous silica might be an excellent adsorbent. Therefore, the adsorption capacity of ordered mesoporous silica was evaluated in comparison with two conventional silicas, ie, Microsilica with a surface area of $78 \mathrm{~m}^{2} / \mathrm{g}$ and N20P with a surface area of $194 \mathrm{~m}^{2} / \mathrm{g}$.

PEG400 has been extensively used in liquisolids as a hydrophilic liquid vehicle due to its nontoxic and nonvolatile properties and excellent dissolving capacity. For liquisolid formulations, poorly water-soluble drugs are firstly dissolved in hydrophilic fluids, and then adsorbed by coating materials, so the drug-loading capacity of the liquisolid formulation can be determined by the amount of adsorbing liquid PEG400 vehicle.

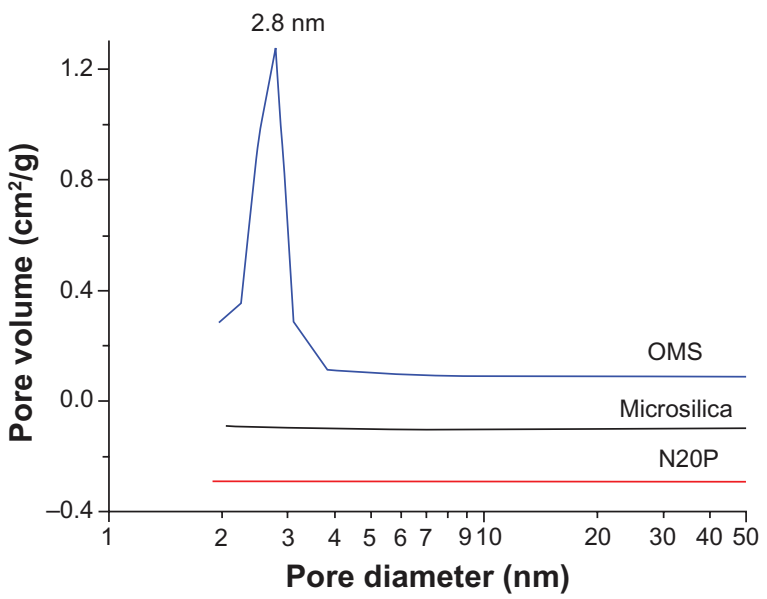

Figure 3 Pore size distribution of ordered mesoporous silica, Microsilica, and N20P.
The measured adsorption capacity of various silicas is compared in Figure 6, which clearly indicates the order of ordered mesoporous silica $>$ N20P $>$ Microsilica. Ordered mesoporous silica with a surface area of $1030 \mathrm{~m}^{2} / \mathrm{g}$ showed an adsorption capacity of $6.9 \mathrm{~g} / \mathrm{g}$, while the adsorption capacity of N20P and Microsilica was $3.9 \mathrm{~g} / \mathrm{g}$ and $2.6 \mathrm{~g} / \mathrm{g}$, respectively. The results indicated that the adsorption capacity was directly related to the surface area of the adsorbent. In addition, the mesoporous structure of ordered mesoporous silica not only contributed to a significantly larger surface area but also generated strong capillary forces to pull liquid into the pores. ${ }^{31-33}$ Therefore, ordered mesoporous silica can hold a larger amount of PEG400 than conventional silica. The adsorption capacity results suggested that the drug liquid loading might be doubled in a liquisolid with ordered mesoporous silica as compared with conventional silicas.

\section{Angle of repose of liquisolid granules}

In the preliminary study, it was noted that flowability of the liquisolid granules was influenced by the ratio of carrier to ordered mesoporous silica. Starch 1500 was chosen as a carrier to mix with the ordered mesoporous silica at various ratios $(4: 1,2: 1$, and $1: 1, \mathrm{w} / \mathrm{w})$ in order to obtain free-flowing liquisolid granules. The angle of repose of the granules indicated that the granules with carrier and ordered mesoporous silica at a ratio of $2: 1(\mathrm{w} / \mathrm{w})$ had the best flowability (Table 3$)$. When the ratio was $1: 1(\mathrm{w} / \mathrm{w})$, the granules broke easily into a fine powder, resulting in poor flow. When the ratio was $4: 1(\mathrm{w} / \mathrm{w})$, the granules adhered together due to insufficient coating material to solidify the liquid. Therefore, a binary mixture of starch 1500 as the carrier and ordered mesoporous silica as the coating material at the ratio of $2: 1(\mathrm{w} / \mathrm{w})$ was selected to form liquisolid system.

\section{Carbamazepine in liquisolid characterized by $\mathrm{x}$-ray diffraction}

Carbamazepine has been found to exist in four different anhydrous crystal polymorphs that may influence its dissolution rate and bioavailability. Further, precipitation of carbamazepine may occur in a liquisolid formulation. Therefore, it is essential to investigate the physical state of carbamazepine in a liquisolid formulation. Figure 7 shows the $\mathrm{x}$-ray diffraction patterns for pure carbamazepine, liquisolid formulations F1 (starch 1500 as carrier), F2 (microcrystalline cellulose as carrier), and the corresponding physical mixtures. Pure carbamazepine was clearly present in a crystalline state because sharp distinct peaks were observed at $2 \theta$ diffraction angles of $13.10^{\circ}, 15.31^{\circ}, 19.50^{\circ}$, and $24.94^{\circ}$ (Figure 7A). 

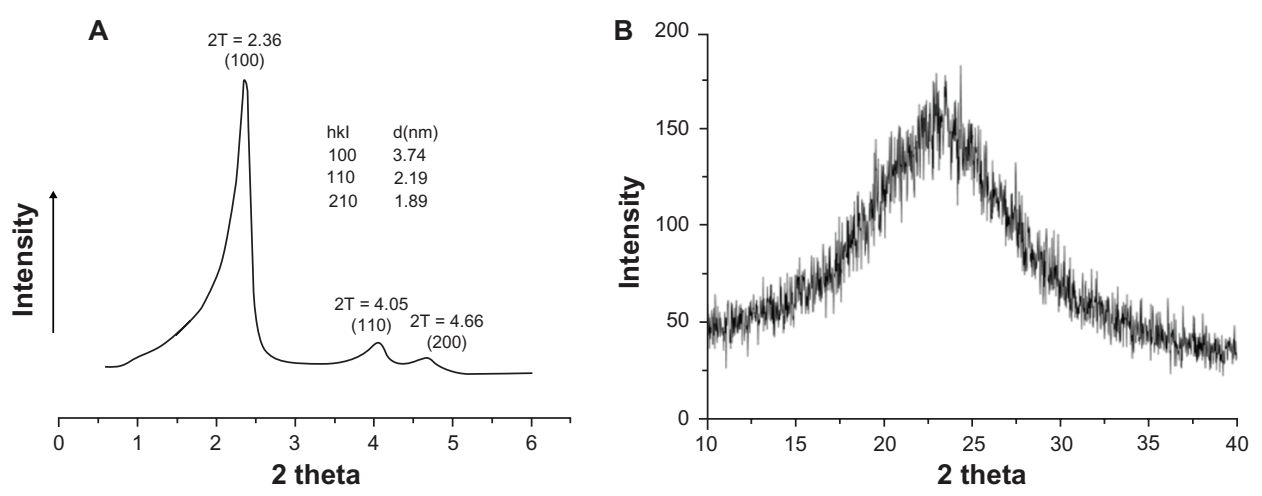

Figure 4 Small-angle x-ray diffraction (A) and wide angle x-ray diffraction (B) patterns of ordered mesoporous silica.

These characteristic carbamazepine peaks were also observed in physical mixtures of F1 and F2 (Figure 7B and D), demonstrating that the crystalline structure of carbamazepine was retained during physical mixing. However, these peaks characteristic of carbamazepine were absent in liquisolid formulations of F1 and F2 (Figure 7C and E), indicating that carbamazepine was almost completely converted from a crystalline to an amorphous or solubilized form by the liquisolid technique. These results are in agreement with the published literature. ${ }^{6,34,35}$ In addition, it was noted that the peaks characteristic of carbamazepine were also absent in the ordered mesoporous silica containing carbamazepine liquid at the concentration used (Figure 7F), indicating that ordered mesoporous silica plays a key role in the amorphous or solubilized form of carbamazepine in a liquisolid formulation. This amorphous or solubilized form of carbamazepine in the liquisolid formulation may also contribute to an improvement in the dissolution rate.

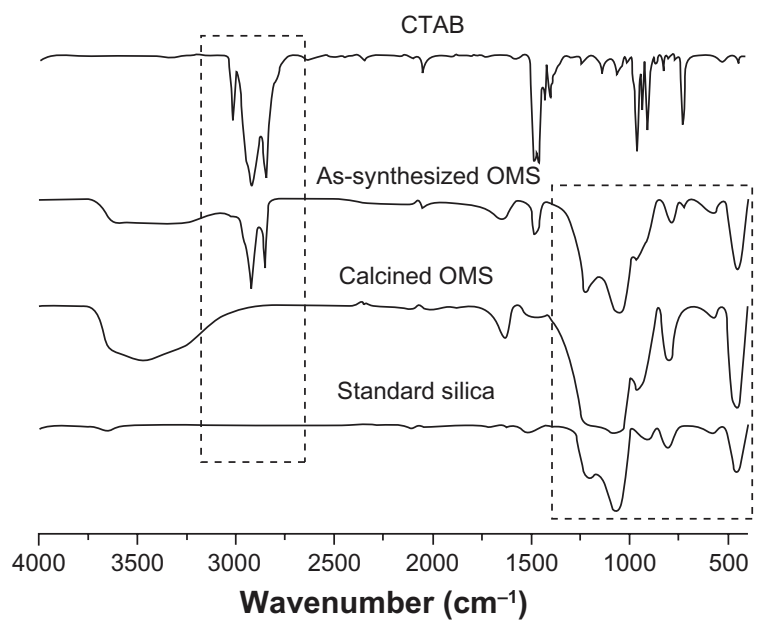

Figure 5 Fourier transform infrared spectra of cetyltrimethyl ammonium bromide (CTAB), as-synthesized ordered mesoporous silica (OMS), calcined ordered mesoporous silica, and standard silica.

\section{In vitro dissolution test}

A dissolution study was performed with the aim of investigating the release behavior of carbamazepine from liquisolid capsules of F1 (starch 1500/ordered mesoporous silica), liquisolid capsules of F2 (microcrystalline cellulose/ordered mesoporous silica), F3 (starch 1500/N20P), carbamazepine powder, and commercially available fast-release carbamazepine tablets. The dissolution profiles in Figure 8 appear to show that liquisolid formulation F1 generated the fastest dissolution rate. After 30 minutes, $86.7 \%$ of carbamazepine was already released from F1, while only $71.7 \%, 69.0 \%$, and $13.6 \%$ of carbamazepine was dissolved from F3, the commercial tablet, and the carbamazepine powder, respectively. The fast release of drug from liquisolid F1 was very likely due to the hydrophilic character of starch and ordered mesoporous silica, the amorphous or solubilized form of carbamazepine in liquisolid formulation, and the huge surface area of ordered mesoporous silica, leading to a large contact area with the dissolution medium.

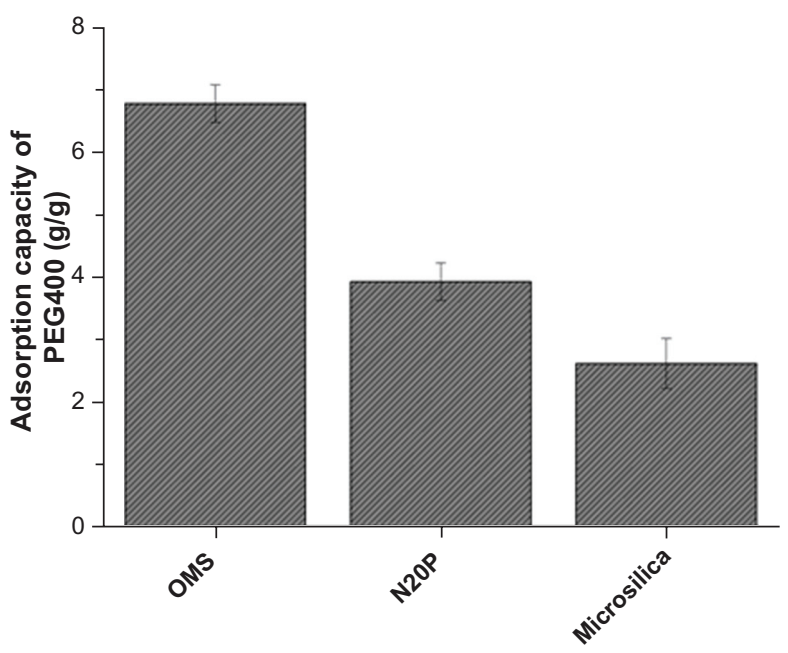

Figure 6 Comparison of adsorption capacity of ordered mesoporous silica (OMS), N20P, and Microsilica $(n=3)$. 
Table 3 Angle of repose of liquisolid granules prepared with various ratios of starch/OMS

\begin{tabular}{ll}
\hline Starch I500:OMS & Angle of repose $\left(^{\circ}\right)$ \\
\hline $1: 1$ & 53.6 \\
$2: 1$ & 42.8 \\
$4: 1$ & 68.9 \\
\hline
\end{tabular}

Abbreviation:OMS, ordered mesoporous silica.

It is important to note that the release of carbamazepine was significantly divergent between the two liquisolid formulations using different carriers, ie, starch 1500 and microcrystalline cellulose. After 60 minutes, more than $90 \%$ of carbamazepine was released from F1 (starch 1500/ ordered mesoporous silica), whereas less than $50 \%$ of the drug released from F2 (microcrystalline cellulose/ordered mesoporous silica) at this time. This clearly indicates that the carrier also played an important role in drug release from the liquisolid formulation. This is in good agreement with previous studies that reported modification of the drug release rate from liquisolids using hydroxypropyl methylcellulose (HPMC), Eudragit, and microcrystalline cellulose as carriers. ${ }^{35-38}$ Compared with liquid-filled drug capsules, the liquisolid capsules exhibited the advantage of easily controlled drug release using suitable carriers.

The marked difference in carbamazepine release between $\mathrm{F} 1$ and F2 suggests that the carrier formed an intact matrix to control drug release after granulation. Because starch is hygroscopic and swellable in water by nature, ${ }^{39}$ the starch matrix was rapidly destroyed when F1 came into contact with water. Compared with starch, microcrystalline cellulose is slightly hygroscopic with a low degree of swelling. ${ }^{40}$ Drug release from F2 was retarded by the matrix of microcrystalline cellulose, resulting in a lower rate of drug release than for F1. Because starch and

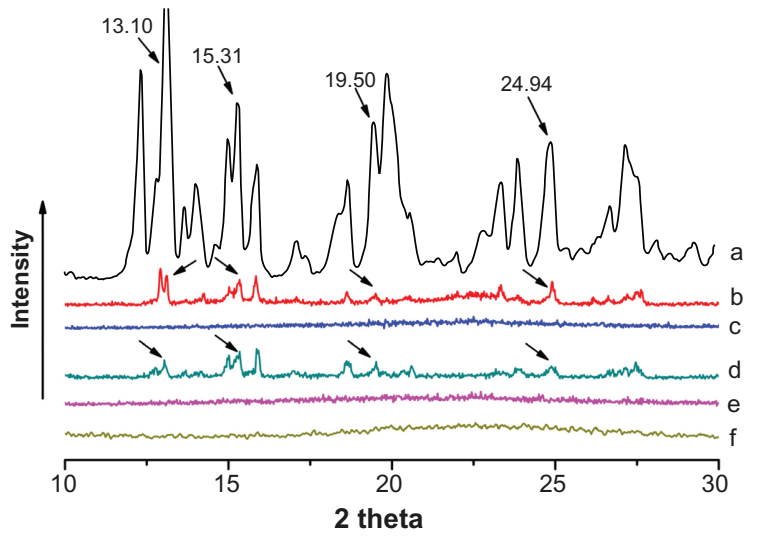

Figure $\mathbf{7}$ Wide angle x-ray diffraction patterns of carbamazepine (A), physical mixture of FI (B), liquisolid FI (C), physical mixture of F2 (D), and liquisolid F2 (E), and carbamazepine liquid in ordered mesoporous silica (F).

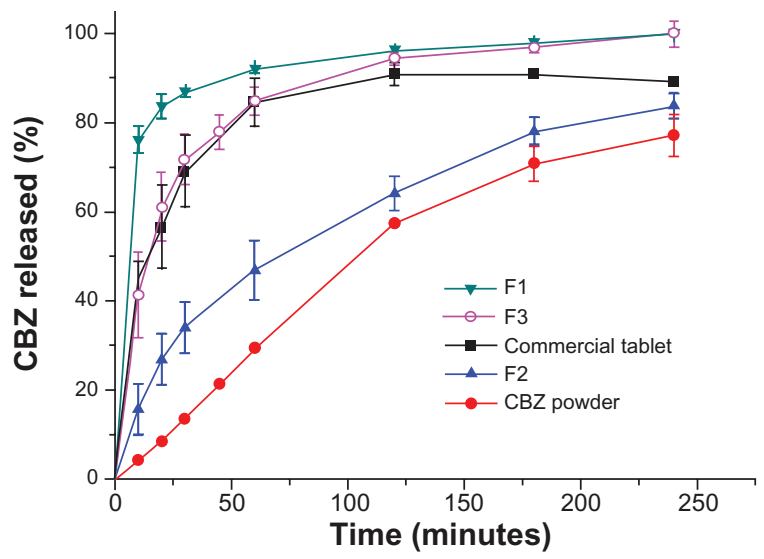

Figure 8 Dissolution profiles of carbamazepine (CBZ) from carbamazepine powder, commercial tablets, prepared liquisolid capsules FI, F2, and F3 $(n=3)$.

microcrystalline cellulose were widely used as carriers in the liquisolid, the findings provide direct experimental evidence for further study of the liquisolid technique.

\section{Pharmacokinetics}

Generally, the bioavailability of carbamazepine, a class II drug according to the biopharmaceutic classification system, is limited by its poor dissolution, and an increase in dissolution rate would result in an improvement in bioavailability. ${ }^{41}$ With the improved dissolution properties of liquisolid capsules using ordered mesoporous silica (F1), an enhancement of pharmacokinetic behavior and bioavailability would be expected to be observable in beagle dogs.

Themean carbamazepine plasma concentration-time profiles following oral administration of the commercial tablets and the prepared liquisolid capsules (F1) are illustrated in Figure 9, and the relevant pharmacokinetic parameters are summarized in Table 4. Oral administration of commercial carbamazepine tablets resulted in mean peak plasma concentration $\left(\mathrm{C}_{\max }\right)$,

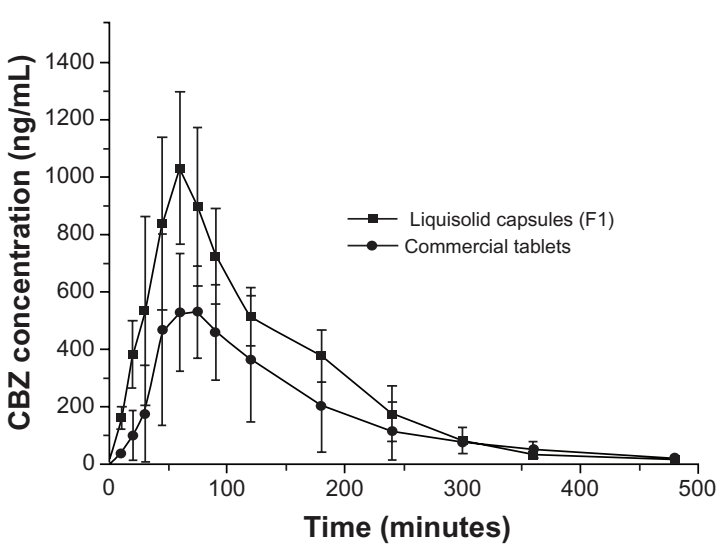

Figure 9 Mean carbamazepine (CBZ) plasma profiles following a single dose, crossover bioavailability study comparing liquisolid capsules with commercial tablets $(n=6)$. 
Table 4 Pharmacokinetic parameters of carbamazepine following oral administration of a single dose ( $100 \mathrm{mg})$ of commercial tablets and prepared liquisolid capsules $(\mathrm{FI}, \mathrm{n}=6)$

\begin{tabular}{|c|c|c|c|}
\hline \multirow[t]{2}{*}{ Pharmacokinetic parameter } & \multirow{2}{*}{$\begin{array}{l}\text { Commercial tablets } \\
\text { Mean } \pm \text { SD }\end{array}$} & \multirow{2}{*}{$\begin{array}{l}\text { Liquisolid capsules }(F I) \\
\text { Mean } \pm \text { SD }\end{array}$} & \multirow[t]{2}{*}{$P$ value } \\
\hline & & & \\
\hline $\mathrm{C}_{\max }(\mathrm{ng} / \mathrm{mL})$ & $669.16 \pm 241.43$ & $1031.44 \pm 266.69$ & 0.05 \\
\hline $\mathrm{T}_{\max }(\min )$ & $69.00 \pm 29.24$ & $60.00 \pm 0.00$ & 0.42 \\
\hline $\mathrm{AUC}_{(0 \rightarrow \infty)}(\mathrm{ng} \cdot$ hour$/ \mathrm{mL})$ & $66357.26 \pm 16549.35$ & $1|7569.4| \pm 9390.1 \mid$ & 0.00 \\
\hline $\mathrm{k}_{\mathrm{e}}\left(\min ^{-1}\right)$ & $0.01 \pm 0.00$ & $0.01 \pm 0.00$ & 0.10 \\
\hline $\mathrm{t}_{1 / 2}(\min )$ & $71.09 \pm 26.32$ & $51.45 \pm 10.93$ & 0.16 \\
\hline
\end{tabular}

Abbreviation: SD, standard deviation.

mean time to peak plasma concentration $\left(\mathrm{T}_{\max }\right)$, and AUC values of $669.16 \pm 241.43 \mathrm{ng} / \mathrm{mL}, 69.00 \pm 29.24$ minutes, and $66357.26 \pm 16549.35 \mathrm{ng} \cdot$ hour/mL, respectively. Meanwhile, following oral administration of liquisolid capsules (F1), the corresponding $\mathrm{C}_{\max }, \mathrm{T}_{\max }$, and AUC values were found to be $1031.44 \pm 266.69 \mathrm{ng} / \mathrm{mL}, 60.00 \pm 0.00$ minutes, and $117569.41 \pm 9390.11 \mathrm{ng} \cdot$ hour $/ \mathrm{mL}$, respectively. Thus, the relative bioavailability was calculated as $182.7 \%(P<0.05)$ for liquisolid F1 compared with the commercial tablet. The in vitro and in vivo studies demonstrated that the liquisolid formulation with ordered mesoporous silica improved not only the dissolution rate, but also the bioavailability of a poorly water-soluble drug.

Moreover, compared with the commercial tablet, enhancement of the oral bioavailability of carbamazepine via the liquisolid formulation suggested that a lower drug dose can be administered using liquisolid capsules to achieve similar clinical effects but minimize the associated adverse effects.

\section{Conclusion}

Ordered mesoporous silica was successfully synthesized as an absorbent material for a liquisolid formulation, and exhibited an excellent adsorption capacity when compared with conventional silicas. The liquisolid formulation prepared using carbamazepine as the model drug, starch as the carrier, and ordered mesoporous silica as the coating material showed significant improvement of in vitro drug release as compared with the commercial fast-release tablet. In vivo testing further demonstrated that the bioavailability of carbamazepine from the orally administered liquisolid preparation was significantly enhanced. As a result, ordered mesoporous silica as a potentially attractive adsorbent may lead to a new approach for development of liquisolid products.

\section{Acknowledgments}

The work was supported by the National Science and Technology Foundation of China (2009ZX09501-023) and the Opening Fund of Sun Yat-sen University Laboratory. The authors thank Mr Yuhai Du for his technical assistance in nitrogen adsorption-desorption measurement.

\section{Disclosure}

The authors report no conflicts of interest in this work.

\section{References}

1. Karmarkar AB, Gonjari ID, Hosmani AH. Liquisolid technology for dissolution rate enhancement or sustained release. Expert Opin Drug Deliv. 2010;7:1227-1234.

2. El-Houssieny BM, Wahman LF, Arafa NM. Bioavailability and biological activity of liquisolid compact formula of repaglinide and its effect on glucose tolerance in rabbits. Biosci Trends. 2010;4:17-24.

3. Nokhodchi A, Aliakbar R, Desai S, Javadzadeh Y. Liquisolid compacts: the effect of cosolvent and HPMC on theophylline release. Colloids Surf B Biointerfaces. 2010;79:262-269.

4. Tiong N, Elkordy AA. Effects of liquisolid formulations on dissolution of naproxen. Eur J Pharm Biopharm. 2009;73:373-384.

5. Mahmoud EA, Bendas ER, Mohamed MI. Preparation and evaluation of self-nanoemulsifying tablets of carvedilol. AAPS Pharm Sci Tech. 2009;10:183-192.

6. Fahmy RH, Kassem MA. Enhancement of famotidine dissolution rate through liquisolid tablets formulation: in vitro and in vivo evaluation. Eur J Pharm Biopharm. 2008;69:993-1003.

7. Tayel SA, Soliman II, Louis D. Improvement of dissolution properties of carbamazepine through application of the liquisolid tablet technique. Eur J Pharm Biopharm. 2008;69:342-347.

8. Spireas S, Sadu S, Grover R. In vitro release evaluation of hydrocortisone liquisolid tablets. J Pharm Sci. 1998;87:867-872.

9. Nokhodchi A, Hentzschel CM, Leopold CS. Drug release from liquisolid systems: speed it up, slow it down. Expert Opin Drug Deliv. 2011;8: 191-205.

10. Friedrich H, Fussnegger B, Kolter K, Bodmeier R. Dissolution rate improvement of poorly water-soluble drugs obtained by adsorbing solutions of drugs in hydrophilic solvents onto high surface area carriers. Eur J Pharm Biopharm. 2006;62:171-177.

11. Kresge CT, Leonowicz ME, Roth WJ, Vartuli JC, Beck JS. Ordered mesoporous molecular sieves synthesized by a liquid-crystal template mechanism. Nature. 1992;359:710-712.

12. Beck JS, Vartuli JC, Roth WJ, et al. A new family of mesoporous molecular sieves prepared with liquid crystal templates. $J$ Am Chem Soc. 1992;114:10834-10843.

13. Kim JY, Yoon SB, Yu JS. Template synthesis of a new mesostructured silica from highly ordered mesoporous carbon molecular sieves. Chem Mater. 2003;15:1932-1934.

14. Zhang WM, Liu J, Sun ZX, Fan BQ, Yang ZD, Forsling W. Synthesis of mesoporous silica by a surface charge reversal route. J Colloid Interface Sci. 2010;349:473-476. 
15. Chen L, Wang YM, He MY. Morphological control of mesoporous silica SBA-15 synthesized at low temperature without additives. J Porous Mater. 2011;18:211-216.

16. He QJ, Cui XZ, Cui FM, Guo LM, Shi JL. Size-controlled synthesis of monodispersed mesoporous silica nano-spheres under a neutral condition. Microporous Mesoporous Mater. 2009;117:609-616.

17. Yu SQ, Zhao RY, Liu CG, Guan C. Triblock copolymer synthesis of mesoporous silica in strong acid medium 1. Characterization of samples and effects of cosolvent and cosurfactant on morphology of mesoporous silica. Abstr Pap Am Chem Soc. 2003;226:U263-U264.

18. Pang XL, Tang FQ. Morphological control of mesoporous materials using inexpensive silica sources. Micropor Mesopor Mat. 2005;85: $1-6$.

19. Langguth P, Kovacevic I, Parojcic J, Homsek I, Tubic-Grozdanis M. Justification of biowaiver for carbamazepine, a low soluble high permeable compound, in solid dosage forms based on IVIVC and gastrointestinal simulation. Mol Pharm. 2009;6:40-47.

20. Elqidra R, Unlu N, Capan Y, Sahin G, Dalkara T, Hincal AA. Effect of polymorphism on in vitro in vivo properties of carbamazepine conventional tablets. J Drug Deliv Sci Technol. 2004;14:147-153.

21. Grzesiak AL, Lang M, Kim K, Matzger AJ. Comparison of the four anhydrous polymorphs of carbamazepine and the crystal structure of form I. J Pharm Sci. 2003;92:2260-2271.

22. Cai Q, Lin WY, Xiao FS, Pang WQ, Chen XH, Zou BS. The preparation of highly ordered MCM-41 with extremely low surfactant concentration. Micropor Mesopor Mat. 1999;32:1-15.

23. Vautier-Giongo C, Pastore HO. Micellization of CTAB in the presence of silicate anions and the exchange between bromide and silicate at the micelle surface: A step to understand the formation of mesoporous molecular sieves at extremely low surfactant and silicate concentrations. J Colloid Interface Sci. 2006;299:874-882.

24. Zana R, Frasch J, Soulard M, Lebeau B, Patarin J. Fluorescence probing investigations of the mechanism of formation of organized mesoporous silica. Langmuir. 1999;15:2603-2606.

25. He QJ, Zhang ZW, Gao Y, Shi JL, Li YP. Intracellular localization and cytotoxicity of spherical mesoporous silica nano- and microparticles. Small. 2009;5:2722-2729.

26. Cejka J, Mintova S. Perspectives of micro/mesoporous composites in catalysis. Catal Rev. 2007;49:457-509.

27. Choy JH, Lee HC, Jung H, Kim H, Boo H. Exfoliation and restacking route to anatase-layered titanate nanohybrid with enhanced photocatalytic activity. Chem Mater. 2002;14:2486-2491.

28. Zhao D, Feng J, Huo Q, et al. Triblock copolymer syntheses of mesoporous silica with periodic 50 to 300 angstrom pores. Science. 1998;279:548-552.
29. Liu K, Feng Q, Yang Y, Zhang G, Ou L, Lu Y. Preparation and characterization of amorphous silica nanowires from natural chrysotile. J Non-Cryst Solids. 2007;353:1534-1539.

30. Wang YD, Zhang S, Ma CL, Li HD. Synthesis and room temperature photoluminescence of $\mathrm{ZnO} / \mathrm{CTAB}$ ordered layered nanocomposite with flake-like architecture. J Lumin. 2007;126:661-664.

31. Ding Y, Mathur A, Chen M, Erlebacher J. Epitaxial casting of nanotubular mesoporous platinum. Angew Chem Int Ed Engl. 2005;44: 4002-4006.

32. Ji QM, Acharya S, Hill JP, et al. Hierarchic nanostructure for automodulation of material release: mesoporous nanocompartment films. Adv Funct Mater. 2009; 19:1792-1799.

33. Wang YM, Wu ZY, Wang HJ, Zhu JH. Fabrication of metal oxides occluded in ordered mesoporous hosts via a solid-state grinding route: The influence of host-guest interactions. Adv Funct Mater. 2006;16: 2374-2386.

34. Tiong N, Elkordy AA. Effects of liquisolid formulations on dissolution of naproxen. Eur J Pharm Biopharm. 2009;73:373-384.

35. Nokhodchi A, Aliakbar R, Desai S, Javadzadeh Y. Liquisolid compacts: The effect of cosolvent and HPMC on theophylline release. Colloids Surf B Biointerfaces. 2010;79:262-269.

36. Javadzadeh Y, Jafari-Navimipour B, Nokhodchi A. Liquisolid technique for dissolution rate enhancement of a high dose water-insoluble drug (carbamazepine). Int J Pharm. 2007;341:26-34.

37. Javadzadeh Y, Musaalrezaei L, Nokhodchi A. Liquisolid technique as a new approach to sustain propranolol hydrochloride release from tablet matrices. Int J Pharm. 2008;362:102-108.

38. Javadzadeh Y, Shariati H, Movahhed-Danesh E, Nokhodchi A. Effect of some commercial grades of microcrystalline cellulose on flowability, compressibility, and dissolution profile of piroxicam liquisolid compacts. Drug Dev Ind Pharm. 2009;35:243-251.

39. Bravo SA, Lamas MC, Salamon CJ. In-vitro studies of diclofenac sodium controlled-release from biopolymeric hydrophilic matrices. J Pharm Pharm Sci. 2002;5:213-219.

40. Builders PF, Bonaventure AM, Tiwalade A, Okpako LC, Attama AA. Novel multifunctional pharmaceutical excipients derived from microcrystalline cellulose-starch microparticulate composites prepared by compatibilized reactive polymer blending. Int J Pharm. 2010;388: 159-167.

41. Emami J. In vitro-in vivo correlation: From theory to applications. J Pharm Pharm Sci. 2006;9:169-189.
International Journal of Nanomedicine

\section{Publish your work in this journal}

The International Journal of Nanomedicine is an international, peerreviewed journal focusing on the application of nanotechnology in diagnostics, therapeutics, and drug delivery systems throughout the biomedical field. This journal is indexed on PubMed Central,

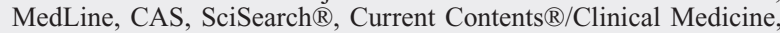

\section{Dovepress}

Journal Citation Reports/Science Edition, EMBase, Scopus and the Elsevier Bibliographic databases. The manuscript management system is completely online and includes a very quick and fair peer-review system, which is all easy to use. Visit http://www.dovepress.com/ testimonials.php to read real quotes from published authors. 